\title{
Observación de un espectro de manifestaciones clínicas ante la exposición a información relacionada con eventos suicidas
}

\section{Observation of a Spectrum of Clinical Manifestations when Exposed to Information Related to Suicidal Events}

\author{
Ricardo Millán-González \\ Universidad de Costa Rica, Costa Rica
}

\author{
Cynthia Córdoba-López \\ Universidad de Costa Rica, Costa Rica
}

\author{
Marjorie Moreno-Salas \\ Caja Costarricense del Seguro Social, Costa Rica
}

\begin{abstract}
Resumen
Hace muchos años se describió el ahora conocido efecto copycat. Se entiende como la reproducción del acto autolesivo, por parte de personas susceptibles, posterior a la exposición a la información en los medios de comunicación relacionada con un suicidio concretado (en noticias, redes sociales, etc.). A partir de la observación en la atención psicológica y psiquiátrica de los pacientes, nuestro grupo ha detectado que esta replicación en realidad forma parte de un espectro mucho más amplio, y que, consecuentemente, otras manifestaciones suelen pasar inadvertidas. Si se trazara un continuum de esas expresiones, en el extremo más severo, se encontraría el suicidio consumado luego de la exposición a dicho material, que es el componente más reconocido hasta la fecha (efecto copycat); no obstante, en otras zonas del espectro, también es posible que las personas experimenten distorsiones cognitivas, componentes obsesivos e intrusivos, ideación pasiva y activa de muerte e intentos no consumados de suicidios. El presente documento se propuso como objetivo describir este fenómeno $\mathrm{y}$, a la vez, ejemplificarlo mediante la exposición de un caso. Resulta probable que el correcto reconocimiento y abordaje fenomenológico sean pasos básicos para establecer los tratamientos y las estrategias preventivas, que garanticen la seguridad de estas personas
\end{abstract}

Palabras clave: suicido, espectro clínico, efecto copycat, secuelas emocionales, medios de comunicación

Ricardo Millán-González y Cynthia Córdoba-López, Universidad de Costa Rica, Costa Rica; Marjorie Moreno-Salas, Caja Costaricense del Seguro Social, Costa Rica.

La correspondencia en relación con este artículo se dirige a Ricardo Millán-González, Universidad de Costa Rica. Dirección electrónica: rmillan@,ClinicaBiblica.com 


\begin{abstract}
:
Long ago, the scientific literature informed about the now well-known copycat effect, understood as a reproduction of the self-injurious act by susceptible individuals after being exposed to suicide-related information in the media (news, social networks, etc.). From observations in psychological and psychiatric care of patients, our group has detected that these manifestations are actually part of a much broader clinical spectrum, where other manifestations can also be found. It would seem plausible that if a spectrum is defined, the most severe end would be consummated suicide, which is the well-recognized component to date (copycat effect); but it is also possible that there is a series of more diverse expressions in other patients after having been exposed to this information, which could include cognitive distortions, obsessive and intrusive phenomena, passive and active ideation of death and unsuccessful suicide attempts. Our purpose with this paper is to explain our observations and present a clinical vignette as an example. We believe that correct recognition and approach to these phenomena is fundamental to establish treatments and preventive strategies that guarantee the safety of this population.
\end{abstract}

Keywords: Suicide, Clinical Spectrum, Copycat Effect, Emotional Sequelae, Media

Los datos de la Organización Panamericana de la Salud (OPS) indican que cerca de 800000 personas al año mueren debido al suicidio. Lo anterior representa el fallecimiento, por esta causa, de alguien cada 40 segundos; de hecho, en personas entre 15 y 29 años constituye la segunda causa de muerte. En el mismo informe, se sugiere que, por cada muerte por suicidio, se presentan 20 intentos de autoeliminación (Comisión Técnica Interinstitucional sobre Estadísticas de Convivencia y Seguridad Ciudadana, 2019).

Ante tan alarmantes datos, Costa Rica ha realizado esfuerzos para seguir los lineamientos de la Organización Mundial de la Salud (OMS) indicados en el Informe Prevención del suicidio. Un imperativo global (Organización Mundial de la Salud, 2014). Así, se ha generado una estructura política y técnica para ofrecer respuesta al fenómeno del suicidio, la cual fue desarrollada en la Política Nacional de Salud Mental y su Plan de Acción para el periodo 2012-2021 (Ministerio de Salud, 2012). Además, se dispone de una Secretaría Técnica en Salud Mental, de reciente creación, adscrita al Ministerio de Salud Pública según se aprobó a través de la Ley 9213 (2014). Desde esta instancia, se ha gestionado la Normativa Nacional para la Articulación Interinstitucional en el Abordaje Integral del Comportamiento Suicida mediante el Decreto N. ${ }^{\circ}$ 40881-S (2018), donde se han planteado los objetivos específicos en este tema. Esta reglamentación estipula que el intento de autoeliminación es de notificación obligatoria para los profesionales en salud mental desde el año 2013. Puntualmente, señala:

Que la Política Nacional de Salud Mental y su Plan de Acción 2012-2021, establecen las prioridades y lineamientos para el abordaje del comportamiento suicida, estableciendo entre sus acciones el desarrollo, formulación e implementación de políticas y acciones de salud mental, dirigidas a personas en riesgo suicida, así como el desarrollo y ejecución de un programa nacional multisectorial para la atención de las personas en riesgo suicida. El Ministerio de Salud mediante el Decreto Ejecutivo No. 37306-S del 27 de agosto de 2012, y ahora recientemente con el Decreto Ejecutivo No. 40556-S del 07 de julio de 2017, "Reglamento de vigilancia de la salud", ordenó 
la notificación obligatoria de todos los "intentos de suicidio", a partir del año 2013. (Decreto N. ${ }^{\circ}$ 40881-S, 2018, p.3)

De acuerdo con los antecedentes de la Política Nacional de Salud Mental y según datos proporcionados por el Ministerio de Salud, durante el período de los años 2000 al 2011, se observa que la tasa de suicidios pasó de 4.7 a 7.3 por cada 100000 habitantes. La tasa ajustada en el 2011 correspondió a un 6.28 por cada 100000 habitantes, para un total de 319 suicidios registrados, que ocurrieron, principalmente, en el grupo de edad que oscila entre los 15 y los 49 años. Se evidenció el predominio de los suicidios en hombres, que representó el $83.38 \%$. Adicionalmente, ese año se reportó un aumento del 7.05\% de defunciones con respecto al mismo periodo anterior (Ministerio de Salud, 2012).

El Ministerio de Salud en el 2013 registró un total de 888 intentos de autoeliminación, mientras que al año siguiente se registraron 1142 intentos, de los cuales el 61.26\% correspondió a mujeres. El mayor porcentaje de casos por grupo de edad fue en personas jóvenes de ambos sexos, entre los 15 y 24 años, lo que representa un 39.02\% del total (Rímola, 2015).

Una revisión del estado del suicidio en Costa Rica indica que la notificación de los intentos de suicidio ha aumentado durante los últimos años, porque en el año 2014 la incidencia era de 25.2 por cada 100 000 habitantes, mientras que el año 2018 esta tasa fue de 41.6 por 100000 habitantes. La población joven presenta picos más altos en estas estadísticas, específicamente las personas de edades entre los 15 y 19 años (Comisión Técnica Interinstitucional sobre Estadísticas de Convivencia y Seguridad Ciudadana, 2019).

Las cifras absolutas de suicidios consumados, aportadas por el Sistema Automatizado de Patología Forense durante este periodo, reportan que en el año 2014 se registraron 288 suicidios ( 238 hombres y 50 mujeres); en el 2015, 394 (259 hombres y 45 mujeres); en el 2016, un total de 343 (291 hombres y 52 mujeres); en el 2017, una cifra de 290 (235 hombres y 55 mujeres) y en el 2018, 327 (274 hombres y 53 mujeres). "En términos porcentuales, tanto en hombres como en mujeres casi el 50\% ocurrió entre los 18 y los 39 años" (Comisión Técnica Interinstitucional sobre Estadísticas de Convivencia y Seguridad Ciudadana, 2019, p.28).

Por cada suicidio consumado, existe un porcentaje mucho mayor, aunque a veces desconocido, de intentos suicidas; esta es una de las razones para invertir en materia de prevención del suicidio. Maris (2002, como se citó en Solano et al., 2016) establece que esta es una tarea compleja, global y obligatoria de abordar, pues, en general, estas acciones son difíciles de desarrollar debido a la naturaleza multidimensional del fenómeno. Esto implica visibilizar la problemática, favorecer los espacios profesionales y seguros para hablar del tema y desmitificar el fenómeno por medio de estrategias de conocida eficacia y eficiencia, delimitadas según los grupos de edad y sus necesidades propias.

Dentro de los aportes en temas de prevención, merece una particular atención el rol de los medios de comunicación y el conocido efecto Werther o copycat, así como su contraparte, el efecto Papageno. El primero se entiende como la consumación de la autoeliminación posterior a que la persona estuvo expuesta a una noticia, donde se narra cómo un individuo cometió un suicidio. A sabiendas de que las personas pueden poseer diferentes recursos de afrontamiento al sufrimiento y malestar psíquico, y que buena parte de la población nacional está expuesta a medios de comunicación que en determinados casos pueden ser sensacionalistas, parcializados y dramáticos, abordar el tema del copycat y otros efectos 
asociados, puede constituirse en un recurso preventivo importante. Precisamente, en esta dirección, el efecto Papageno plantea que cubrir una noticia de esta índole de manera responsable, al exponer información sobre las personas que, en situaciones críticas, han afrontado la experiencia de forma positiva y sin conductas suicidas, puede tener un efecto protector (Herrera et al., 2015).

El sociólogo norteamericano D.P. Phillips acuñó el término “efecto Werther", en 1974, para referirse a la sugestión de la conducta suicida. Esto lo toma del fenómeno asociado a la novela "The sorrow of young Werther" (Las penas del joven Werther), publicada en 1774 y escrita por el alemán Johan W. von Goethe. Esta historia narra las desesperanzas amorosas de un joven talentoso, quien muere por dispararse en la cabeza. La venta de este libro llegó a ser prohibida en varios lugares de Europa, porque se suscitaron numerosos decesos de jóvenes mediante el mismo método (Pérez, 2019).

En ese trabajo pionero, Philips (1974) señaló las implicaciones del efecto Werther. Explicó que la sobreexposición a la información asociada con un suicidio de una persona famosa o un personaje público tiende a desencadenar la imitación del mismo acto.

Pirkis y Blood (2010), citados por Álvarez, (2012), analizaban 97 investigaciones relacionadas entre los medios de comunicación, la prensa, la radio y la televisión, junto con internet y un desenlace suicida. "Se trataba de una multitud de casos centrados, en su mayoría, en noticias publicadas en medios de prensa; este análisis encontró una relación causal evidente entre los aumentos en el número de suicidios y la influencia de la prensa" (p.49).

En 2003, Gould et al. (citados por Álvarez, 2012), postularon lo siguiente: "ya no queda ninguna duda respecto a la validez de los efectos contagiosos del suicidio", tras analizar 42 reportes de prensa, de los cuales 29 (el 69\%) tenía una evidente influencia en suicidios por imitación (Álvarez, 2012, p. 49).

La evidencia científica, en torno a la imitación de la conducta autolesiva, ha llevado a la OMS y la OPS (Organización Mundial de la Salud [OMS]-Organización Panamericana de la Salud [OPS], 2018) a indicar que los medios de comunicación suministran una amplia gama de información; por lo tanto, influyen fuertemente sobre las actitudes, las creencias y los comportamientos de la comunidad, así como en la política, la economía y la práctica social. Consecuentemente, estos recursos podrían desempeñar un papel preponderante en la prevención del suicidio, sobre todo, porque, la transferencia global de la información, hoy se presenta a partir de las diferentes vías de comunicación: las series televisivas, las películas, el internet, las redes sociales, la prensa y la televisión.

Lo anterior presupone un reto aún más complejo en cuanto a la prevención del suicidio, porque existen otras categorías de acceso a esa información que escapan de la posibilidad de monitoreo y, por lo tanto, de normas éticas y morales. Ruder et al. (2011) indican que diversos foros y sitios web dedicados al suicidio pueden, incluso, animar activamente las conductas autolesivas de sus usuarios. Sin embargo, controlar y regular estos espacios conlleva limitaciones importantes, pues responden a la legislación y la normativa propia de cada país, entre otros factores.

Dentro de los elementos retadores para prevenir el efecto copycat, más allá de la discusión del medio que comunica o pone a disposición la información, se relaciona con cuál es la población de mayor vulnerabilidad y el procesamiento de la información llevado a cabo por los diferentes grupos 
poblacionales. Ju Ji et al. (2014) analizaron las cualidades de los informes mediáticos y el efecto de imitación en función de variables sociodemográficas tales como la edad, el sexo y el método suicida. Su estudio concluye que existe un efecto de imitación en la comunidad, después de los reportajes intensivos e indiscriminados de un suicidio de alguna celebridad. Esto se presenta en ambos sexos, pero es más frecuente en el subgrupo de edades más jóvenes o similares a la de la persona fallecida; además, tiende a replicarse el mismo método suicida.

El estudio de Cheng et al. (2007) propone otros factores de riesgo tales como el estado clínico de la depresión mayor y una historia previa de comportamiento suicida. Ellos reportan un aumento del riesgo de dos veces entre los sujetos con pensamientos suicidas anteriores y de hasta de tres veces entre los que tenían intentos previos de autoeliminación.

El estudio realizado por Ju Ji et al. (2014), al consultar diversas investigaciones, anota la importancia de las variables demográficas (por ejemplo, el género y la edad) en la población vulnerable. Sin embargo, parece ser que la diferencia entre la imitación y la significancia de estas variables estaría asociada también a la diferencia entre la calidad y el acceso a la información sobre el suicidio.

Swanson y Colman (2010), por su parte, encontraron que la exposición al suicidio predice la replicación del acto; esto ocurrió en todos los grupos de edad, aunque el riesgo fue mayor en los más jóvenes. Los tipos de exposiciones evaluadas (el suicidio de un compañero de la escuela y el hecho de conocer personalmente a quien falleció) predijeron la replicación del acto al ser mayor la posibilidad en el primer caso. También, hubo una mayor tendencia a los intentos autolesivos y, en menor grado, a la ideación suicida. Asimismo, encontraron que, en estos casos, el riesgo de muerte fue independiente de la presencia de ansiedad o depresión previa, el apoyo social y la relación con la persona que falleció.

La investigación y el análisis de los diversos autores citados se ha centrado únicamente en la atención o la prevención de la imitación de la conducta suicida ante el manejo de la noticia. El presente documento contiene un carácter propositivo a nivel teórico, en tanto describe un espectro de manifestaciones clínicas ante la exposición a información relacionada con eventos suicidas que Millán (2016) ha sistematizado y el resto del equipo de investigación ha confirmado en su práctica profesional.

Además, el polo extremo del espectro planteado teóricamente se ejemplifica con un estudio de caso, en el cual una paciente sufrió un impacto afectivo ante la noticia del suicidio de una persona reconocida públicamente; luego de este evento, esta persona inició con ideación suicida por primera vez en su vida.

\section{Desarrollo}

Al procurar una línea diferente de estudio, el presente artículo pretende problematizar, desde otra arista, el tema en cuestión. Nuestra intención consiste en plantear y discutir la gama de expresiones clínicas en las personas que recibieron una noticia sobre un suicidio consumado y no limitarnos específicamente a la descripción de las características de los casos donde hubo una replicación del acto.

Así, nuestra pregunta clínica es la siguiente: ¿existe un espectro de respuestas más amplias que anteceden a la imitación del acto autolesivo o que forman parte, en sí mismas, de manifestaciones clínicas 
distintas a la consumación del acto y que se presentarían en otros grupos de personas, que se vieron expuestas a la noticia de un suicidio?

En esta línea, Millán (2016) ha descrito un espectro más extenso, en donde las semanas posteriores a la difusión de noticias de actos suicidas se presenta un aumento en el número de expresiones emocionales y cognitivas en los pacientes, las cuales fueron generadas específicamente por la exposición a esa información. A partir de la observación en la atención psicológica y psiquiátrica de los pacientes, nuestro grupo ha confirmado esta gama de manifestaciones, que trascienden la replicación de la conducta autolesiva. Así, cuando la severidad se define en función de la ideación/ejecución del acto suicida, en el extremo más severo, se encontraría el suicidio consumado, que, como bien se ha descrito en este documento, es el componente más reconocido hasta la fecha. También, resulta posible que exista una serie de exteriorizaciones más diversas en un grupo de pacientes. A continuación, se presenta la descripción detallada de lo que hemos denominado "el espectro de las manifestaciones clínicas por la exposición a información relacionada con eventos suicidas", y que se sustenta en lo que hemos observado en un grupo de pacientes a los que les hemos brindado una atención clínica. La representación esquemática se expone en la Figura 1.

Figura 1

Espectro clínico por la exposición a información sobre eventos suicidas

Algunos factores protectores: grupq de apoyo efeqtivo, buena adaptación premórbida y recursos emocionales adaptativos y sanos, disponibilidad de intervenciones psicológicas/psiquiátricas efectivas
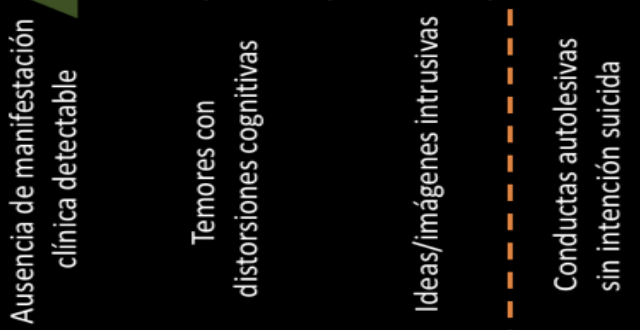

I
I
I
I
I
I
I
I
I
I
K
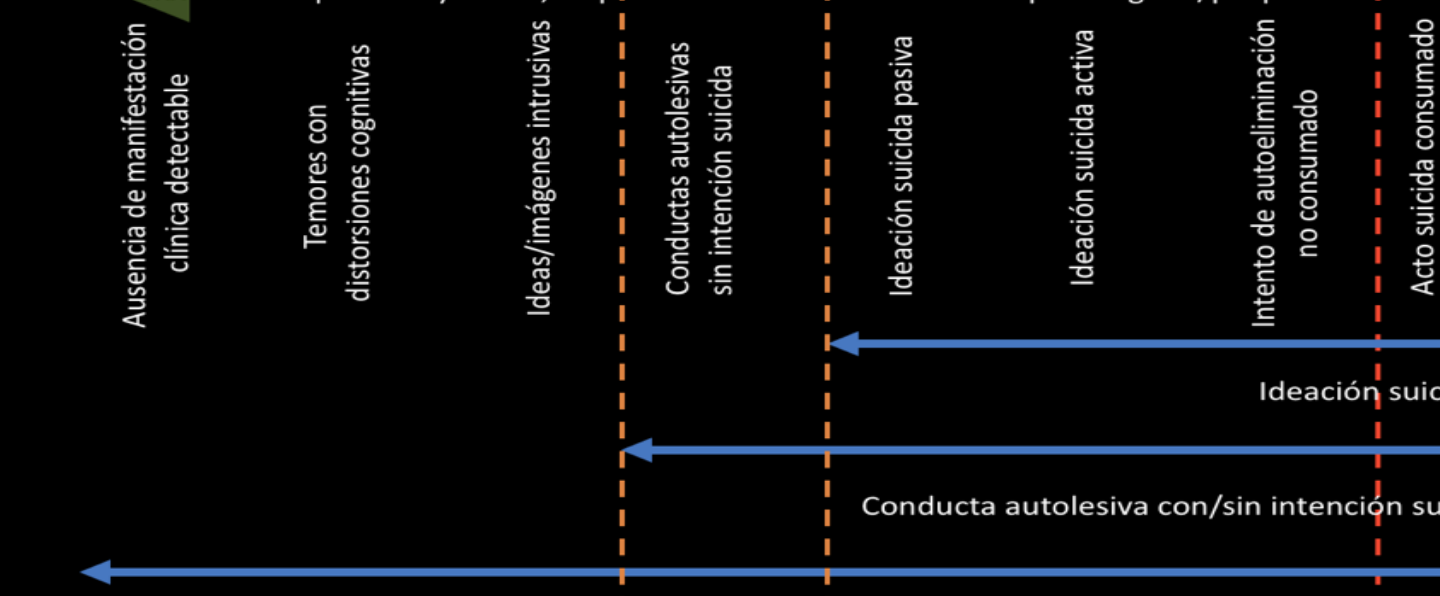

Menor conducta/ideación suicida

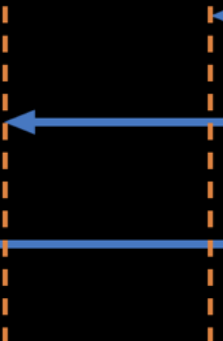

Ideación suicida

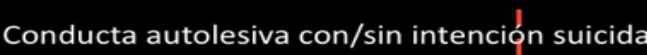

Algunos factores de riesgo: carencia de un grupo de apayo efectivo, pobre adaptación premórbida, recursos emocionales desadaptativos e inmaduros, ausencia de intervenciones psicológicas/psiquiátricas efectivas

Fuente: elaboración propia. 
El fenómeno consiste en ocho categorías de manifestaciones clínicas:

1. Ausencia de sintomatología detectable: en el extremo más distante del suicidio consumado (lado izquierdo de la figura) estarían las personas sin expresiones psicopatológicas por la exposición al material en cuestión. En ellas es viable que no existan elementos detectables en donde haya afectación emocional o cognitiva. Creemos que la mayoría de las personas, quienes reciben esta información, formarían parte de este grupo.

2. Temores o distorsiones cognitivas: en ocasiones, los pacientes consultan o expresan angustia a que una condición psiquiátrica, diagnosticada previamente o no, evolucione de tal forma que ellos mismos cometan un acto suicida más adelante en sus vidas; esto se presenta sin que exista una idea pasiva o activa de muerte. Con frecuencia, se trata de una consecuencia del desconocimiento sobre su propio padecimiento, o porque los medios de comunicación han propiciado una serie de estigmas sobre la condición mental; ello sucedería a partir de presentar la información mediante generalizaciones absolutas, sin un sustento científico o en ausencia de apego a los lineamientos de la OPS/OMS (2018), en cuanto a la publicación de noticias asociadas con suicidios. De esta manera, algunos usuarios exponen distorsiones cognitivas en donde suponen que "todas las personas con una condición psiquiátrica (independientemente de sus características) evolucionan en severidad hasta atentar contra sus vidas". De ahí, consecuentemente, se desprenden emociones desbordadas y conductas desadaptativas de distintos tipos.

3. Ideas e imágenes intrusivas: en otros casos, el malestar se experimenta en forma de ideas intrusivas con imágenes o pensamientos sumamente angustiantes para ese individuo, lo que bien se puede clasificar dentro de las manifestaciones postraumáticas. Así, estas personas podrían experimentar escenas de verse a sí mismos fallecer de la misma manera que murió el sujeto expuesto en la noticia, o la idea de que si se pasa por el mismo lugar donde se consumó el suicidio (por ejemplo, por precipitación desde un puente), el paciente puede perder el control de sí mismo e incurrir en la misma conducta (por ejemplo, al lanzarse del puente). Típicamente, estos eventos generan un alto nivel de malestar. Impiden efectuar traslados o mantener un adecuado nivel de funcionamiento en las diversas áreas de la vida de esa persona. Nuevamente, en estos casos, es usual que no se identifiquen ideas pasivas o activas de muerte.

4. Conductas autolesivas sin ideación suicida: si se avanza en la severidad del espectro con respecto al componente ideación/acto suicida, algunos pacientes podrían incurrir en conductas autolesivas sin intención suicida, tal y como lo describe el Manual Diagnóstico y Estadístico de los Trastornos Mentales (DSM-5) (American Psychiatric Association [APA], 2014). En estos casos, la angustia o el malestar generados por la exposición a la noticia intensificaría una condición de fondo; así, si existen recursos emocionales inmaduros, la persona expuesta podría incurrir en daños autogenerados sin intención de muerte como una forma de lidiar con el dolor generado por la información.

5. Ideación suicida pasiva: en algunas ocasiones, los pacientes podrían iniciar con ideas de muerte pasivas, luego de la exposición a la noticia. Típicamente, son personas que mantienen sintomatología depresiva o ansiosa activa, aunque no siempre detectada. En estos casos, el paciente presentaría un 
incremento en el nivel de desesperanza, usualmente, si hay identificación con la persona fallecida y, por lo tanto, manifestaría pensamientos o fantasías de "no estar con vida" mediante actos fortuitos, tales como accidentes de diversa índole. Es usual que estas personas mantengan un buen nivel de funcionamiento premórbido y que cuenten con diversos recursos personales o de red de apoyo, que finalmente limitarían la progresión de esta manifestación sintomática.

6. Ideación suicida activa: más adelante, en el espectro, hay personas que empezarán con ideas de muerte activa, luego de la exposición. Muchos de ellos podrían experimentar ideas de cómo atentar contra su vida y, eventualmente, se estructuraría un plan de acción. En caso de que exista alguna intervención psicológica o farmacológica, una buena respuesta del grupo de apoyo o una adecuada estructuración de sus mecanismos de defensa, la progresión clínica podría limitarse.

7. Intento de autoeliminación no consumado: si el nivel de desesperanza aumenta aún más por la alta identificación con la persona fallecida, por afectaciones en los mecanismos adaptativos emocionales, por la carencia de un grupo de soporte, por la ausencia de una intervención efectiva o por la severidad de la enfermedad psiquiátrica de fondo, de aquí se desprenderá el subgrupo de personas que ejecutarán los actos suicidas fallidos y, también, los consumados. En ambos casos, es usual que haya abuso de alcohol u otras sustancias. La letalidad del método elegido, la impulsividad y el uso comórbido de sustancias serían algunos de los factores diferenciadores del desenlace. El primer grupo podría no ser detectado en caso de que no haya una consulta al personal de salud, o bien se asociaría con el incremento en la atención de intentos de autoeliminación, que se ha observado luego de la exposición a noticias con las características descritas; esto es más visible para el personal que labora en servicios de urgencias a nivel psiquiátrico o en hospitales generales.

8. Intento de autoeliminación consumado: finalmente, como es ampliamente reconocido, hay un grupo de personas minoritario que cometerán el suicidio y son usualmente los captados por las estadísticas de los sistemas de salud; por lo tanto, corresponden a los casos más descritos en la literatura científica. Es viable que en esta sección predominen los hombres, las personas con psicopatología sin tratamiento o descompensada, el consumo de sustancias y la tendencia a la impulsividad.

Se aclara que las manifestaciones expuestas son generales y es viable que existan otros fenómenos que se incluirían dentro del espectro descrito. Además, resulta posible que no exista una evolución lineal entre las fases y que una expresión clínica fluctúe entre los componentes descritos sin una secuencia lógica. Algunas variables, que impactarían en este proceso, son los conflictos internos o vinculares específicos y la sobrecarga de estresores psicosociales. Muchos datos parecieran indicar que los sobrevivientes de suicidio, o bien, quienes recientemente han llevado a cabo un acto autolítico, junto con quienes presentaban la ideación suicida días antes, pueden agudizar la sintomatología derivada de la exposición a la noticia e impactar, de forma negativa, su calidad de vida y agregar factores de riesgo al proceso de cada individuo.

También, se enfatiza en el hecho de que la presente descripción considera los componentes afectivos de la enfermedad depresiva o ansiosa, así como del abuso de sustancias; es posible, sin embargo, que 
dentro de las manifestaciones psicóticas o de otros tipos, existan repercusiones asociadas con el espectro mencionado. Además, puede ocurrir, en casos de diagnósticos de trastornos de la personalidad graves, que los acting out y las estrategias desadaptativas puedan presentarse desde conductas impulsivas, o bien marcadas fluctuaciones anímicas.

Un apartado independiente lo merecen los familiares de quienes consumaron el suicidio y que, al ver la exposición de su ser querido en medios de comunicación, podrían experimentar, además de los cuadros descritos, emociones de intenso enojo, furia, desamparo y molestia por el trato que se le prorporciona a esta información sensible. Las respuestas específicas de estas personas estarán dictadas por los mecanismos adaptativos de los que dispongan.

\section{Un ejemplo de la presentación clínica de la quinta categoría: ideación suicida pasiva}

Con el fin de ilustrar uno de los elementos del espectro, que prevalece en la consulta y demanda intervenciones específicas a los clínicos, se presenta, a continuación, un estudio de caso. Se conceptualiza de la siguiente forma: "Es una técnica de investigación cualitativa que tienen como característica prestar especial atención a cuestiones que específicamente pueden ser conocidas a través de casos. El caso puede ser simple o complejo: una persona, un grupo o una institución” (Ortiz, 2003, p.61).

El tipo de estudio de caso, que se presenta en esta oportunidad y la preponderancia que ocupa dentro del planteamiento, se indica de acuerdo con el siguiente argumento:

El estudio instrumental de casos se propone analizar para obtener una mayor claridad sobre un tema o aspecto teórico (por lo tanto, el caso concreto es secundario). El caso es un instrumento para conseguir otros fines indagatorios. Se puede dar cuando el investigador selecciona intencionalmente un caso porque busca un objetivo más allá del mismo. Por ejemplo, para ilustrar un problema, una temática o un argumento (Sabariego et al., 2009, p.314).

Los datos de la persona fueron recopilados por medio de una entrevista semiestructurada (la persona entrevista brindó su consentimiento para participar como caso de estudio con fines de investigación). Para ello, se empleó una guía de preguntas, que se agruparon por variables de interés, tales como conocer los antecedentes clínicos de la paciente, la historia de vida asociada al tema de suicidio, la identificación con el evento, el tipo de vinculación con el fallecido, el efecto posterior a la noticia, la presencia de sintomatología y las estrategias de afrontamiento empleadas por la entrevistada.

Una vez obtenida la información, se realizó un análisis de contenido de índole cualitativo. En primer lugar, se identificó el tema de análisis (efecto de la noticia de suicidio). Posteriormente, se determinaron las categorías de análisis que fueron codificadas según su presencia o ausencia. Entre estas pueden citarse las siguientes: presencia de cuadro clínico previo a la noticia de suicidio, la historia de suicidios cercanos, la identificación con la persona fallecida, la respuesta sintomatológica posterior a la noticia y la forma de afrontar el evento. Este análisis del caso presentado ayuda a confirmar las manifestaciones clínicas observadas en otros pacientes, quienes han compartido experiencias similares ante la noticia de suicidio de una persona que admiran mediante los medios de comunicación. 
A continuación, el resumen de interés:

Mujer profesional de mediana edad quien, previo a la presencia de sus manifestaciones clínicas, estuvo expuesta a dos noticias relacionadas con el suicidio: la primera de un personaje mundialmente reconocido y por quien sentía una cuantiosa admiración y la segunda, una comunicación polémica, a nivel nacional, debido a un comentario sobre el fenómeno del suicidio en redes sociales por parte de una figura política costarricense.

Durante la entrevista retrospectiva, se detectó un episodio depresivo mayor, que previamente no había sido identificado, el cual se caracterizó por más de dos semanas de presentar lo siguiente: sentimientos persistentes de tristeza, anhedonia, baja autovaloración, descenso en el nivel de energía, distractibilidad e ideas obsesivas recurrentes. Luego de que la paciente se expuso a los dos eventos descritos, ella inició, por primera vez en su vida, con ideas pasivas de muerte, las cuales consistían en el deseo de "no estar con vida", o que "ocurriera un accidente aéreo" en donde ella falleciera.

En lo descrito, se evidencia cómo el caso presentado ilustra la sintomatología descrita en el quinto punto del espectro graficado en el apartado anterior; es decir, presencia de ideas pasivas de muerte, sintomatología del espectro depresivo, un buen nivel de funcionamiento premórbido, y con diversos recursos personales, y redes de apoyo que pueden asociarse a un limitación en la progresión de esta manifestación sintomática.

\section{Discusión}

Tal y como ha sido expuesto previamente, Costa Rica mantiene unas estadísticas de suicidio alarmantes, sobre todo, al considerar que, en promedio, fallece una persona al día por esta causa. De ahí, la importancia que adquiere el sistema nacional de respuesta y atención a esta problemática. En ese sentido, se trata de poblaciones sumamente diversas, de características particulares, pero concentradas en la población adulta joven, con mayor letalidad en los hombres y más intentos de autoeliminación en las mujeres.

A pesar de que la OMS ha emitido recomendaciones para el manejo de estos temas en los medios de comunicación, no siempre las noticias cumplen con dichas sugerencias y la información puede ser transmitida en una línea que propicie el efecto copycat. Consideramos que, consecuentemente, luego de un suicidio que sea expuesto en medios de comunicación, el personal sanitario deberá mantenerse alerta e indagar activamente sobre los componentes descritos en el presente documento.

La prevención de este fenómeno contiene muchas aristas. La evidencia muestra que los medios de comunicación juegan un papel potenciador o protector, según sea el manejo que se formule. Nuestro equipo ha constatado, a nivel pragmático, que las personas pueden experimentar diferentes manifestaciones cognitivas, somáticas y afectivas, al encontrarse expuestas a noticias de suicidio. Nuestra observación clínica comprueba que existen, por lo tanto, múltiples expresiones que trascienden la consumación del acto, todas las cuales podrían, eventualmente, ser reconocidas y atendidas por el personal sanitario cuando se presentan las circunstancias descritas.

En el caso específico del caso clínico descrito, en función de lo propuesto sobre la observación de un espectro de manifestaciones clínicas, al considerar una afectación a nivel intermedio dentro del espectro, se identificó que la exposición a las noticias señaladas desencadenó ideas intrusivas de muerte 
con la consiguiente elaboración de ideas pasivas de muerte, desregulación afectiva, hipersomnia, falta de energía, pérdida de apetito y desesperanza; es decir, se intensificó la sintomatología de un episodio depresivo ya existente. Cabe señalar que la entrevistada se autodescribió como "muy admiradora de la persona mundialmente famosa" que cometió el suicidio; ella se identificaba con esta celebridad de muy distintas maneras y era seguidora de sus programas.

Consideramos que en una persona susceptible (en este caso, con sintomatología depresiva no identificada previamente), con un vínculo emocional y de admiración por quien ejecuta el suicidio, y luego de un comentario polémico por parte de una figura pública en donde se expuso el acto como digno de valentía, se encuentra la suficiente justificación para explicar la intensificación del estado depresivo. Como parte de él, es viable que hayan surgido, por primera vez en la vida de esta paciente, una ideación pasiva de muerte. Es nuestra apreciación que, gracias a sus recursos personales, así como a una atención profesional oportuna y temprana en el proceso, el cuadro clínico no evolucionó. De esta manera, no se presentó un avance hacia el extremo de mayor severidad dentro del espectro de las manifestaciones clínicas por la exposición a información relacionada con eventos suicidas (ideación suicida activa, intento de autoeliminación no consumado).

Una vez reconocida toda la fenomenología descrita en el presente documento, estaría pendiente definir cuáles pacientes viven estos eventos de forma aislada y, por lo tanto, no necesariamente evolucionarían hacia conductas autolesivas consumadas; también, sería oportuno ubicar los usuarios que sí acarrean un mayor riesgo y, consecuentemente, ameritan más cuidados y tratamientos. Otra labor básica para el futuro sería estructurar las herramientas de tamizaje más adecuadas para los pacientes que forman parte del espectro y reciben una atención médica, además de estratificar las intervenciones según el riesgo de cada persona.

Consideramos que es valioso observar la eventual existencia y el subregistro de las repercusiones humanas y emocionales posteriores a la exposición de noticias de un suicidio consumado. Prestar atención a otras manifestaciones clínicas, y generar una sistematización de las mismas, podría ampliar las descripciones, o bien facilitar las intervenciones en grupos vulnerables. Además, creemos que merece una particular atención la población de adolescentes, por ser más susceptibles a la imitación, al igual que el grupo de adultos jóvenes, quienes encabezan las estadísticas de suicidio consumado en nuestro medio. De igual manera, vale la pena definir si se requieren acciones diferenciadas entre hombres y mujeres, debido a la mayor letalidad de los actos suicidas en los primeros, así como de los intentos no consumados en las segundas.

Se esperaría que, en el futuro, los profesionales en salud, al partir del presente espectro, puedan con mayor agilidad orientar las intervenciones respectivas, en especial el personal de Psiquiatría y Psicología clínica. El enfoque de la literatura sobre el fenómeno del copycat se ha hecho sobre la prevención, pero, quizás, ha dejado de lado que la misma iniciaría al definir quién ingresa dentro del cuadro clínico descrito; así, se beneficiarían las personas con riesgo suicida y todas aquellas con riesgo únicamente de morbilidad psiquiátrica. También, esperaríamos que estas consideraciones fueran incluidas, eventualmente, y luego de una mayor sistematización de los hallazgos en los programas sobre prevención del suicidio existentes en nuestro medio. 


\section{R. Millán-GonzÁlez, C. Córdoba-LóPez y M. Moreno-Salas}

Además del dolor humano para cada uno de los pacientes y sus familiares, afrontar estas situaciones genera posibles aumentos en los costos de atención hospitalaria, trátese de servicios de hospitalización o consulta externa, a nivel público o privado. Asimismo, los servicios de emergencias pueden impactarse debido a un aumento en las consultas durante los días posteriores a la presentación de la información. Algunas de las personas podrían requerir incapacidades laborales para recuperarse del desequilibrio emocional. Si se definen protocolos o acciones anticipadas, la atención en estas circunstancias podría ser más eficiente y efectiva, lo que permitiría una mejor distribución de los recursos económicos y humanos.

\section{Referencias}

Álvarez, S.M. (2012). Efecto Werther: Una propuesta de intervención en la facultad de Ciencias Sociales y de la Comunicación (UPV/EHU). Norte de salud mental, X(42), 48-55. https://dialnet.unirioja.es/descarga/ articulo/3910960.pdf

American Psychiatric Association (APA). (2014). Manual Diagnóstico y Estadístico de los Trastornos Mentales DSM-5. Editorial Médica Panamericana.

Cheng, A., Hawton, K., Chen, T., Yen, A., Chang, J., Chong, M., Liu, C., Lee,Y., Teng, P., \& Chen, L. (2007). The influence of media reporting of a celebrity suicide on suicidal behavior in patients with a history of depressive disorder. Journal of Affective Disorders, 103, 69-75. https://doi.org/10.1016/j.jad.2007.01.021

Comisión Técnica Interinstitucional sobre Estadísticas de Convivencia y Seguridad Ciudadana (2019). Estado del suicidio en Costa Rica. Periodo 2014-2018. COMESCO.

Decreto N. ${ }^{\circ} 40881$-S Oficialización de la Normativa Nacional para la Articulación Interinstitucional en el Abordaje Integral del Comportamiento Suicida (2018). La Gaceta, Alcance $N^{\circ} 38$, Poder Ejecutivo, Decretos, 22 de febrero de 2018, 20-71. https://www.imprentanacional.go.cr/pub/2018/02/22/ALCA38_22 02_2018.pdf

Herrera, R., Ures, M., \& Martínez, J. (2015). El tratamiento del suicidio en la prensa española: ¿efecto werther o efecto papageno? Revista De La Asociación Española De Neuropsiquiatría, 35(125), 123-134.

Ju Ji, N., Young Lee, W., Seok Noh, M., \& Yip, P. (2014). The impact of indiscriminate media coverage of a celebrity suicide on a society with a high suicide rate: Epidemiological findings on copycat suicides from South Korea. Journal of Affective Disorders, 156, 56-61. http://dx.doi.org/10.1016/j.jad.2013.11.015

Ley 9213 Creación de La Secretaría Técnica de Salud Mental, Modificación de La Ley No 5412, Ley Orgánica del Ministerio de Salud, y sus reformas; reformas, y adición de un párrafo al inciso C) del Artículo 8 de la Ley $\mathrm{N}^{\mathrm{o}} 8718$, Autorización para el cambio de nombre de la Junta de Protección Social y establecimiento de la distribución de rentas de las loterías nacionales, y sus reformas (2014). La Gaceta, $N^{\circ}$ 69, Poder Legislativo, Leyes, 8 de abril de 2014, 1-4. https://www.imprentanacional.go.cr/pub/2014/04/08/COMP 08 04_2014. $\underline{\text { html\# Toc384650194 }}$

Millán, R. (16 de septiembre de 2016). Mucho más que el efecto 'copycat' del suicidio. La Nación. https://www. nacion.com/opinion/foros/mucho-mas-que-el-efecto-copycat-del-suicidio/7KYJUYMCTVAEDGX53JXH LPBN4Q/story/

Ministerio de Salud. (2012). Política Nacional de Salud Mental 2012-2021. Ministerio de Salud. 
OBSERVACIÓN DE UN ESPECTRO DE MANIFESTACIONES CLÍNICAS

Organización Mundial de la Salud. (2014). Prevención del suicidio: un imperativo global. Organización Panamericana de la Salud. http://apps.who.int/iris/bitstream/10665/136083/1/9789275318508 spa.pdf

Organización Mundial de la Salud-Organización Panamericana de la Salud (2018). Prevención del suicidio un instrumento para profesionales de los medios de comunicación. Actualización del 2017. https://iris.paho. org/bitstream/handle/10665.2/49121/OPSNMH180021_spa.pdf?sequence=1\&isAllowed=y

Ortiz, F. (2003). Diccionario de metodología de la investigación científica. Limusa.

Pérez, S.A. (2019). Los medios de comunicación y el suicidio. Evidencias y Sugerencias. https://www.redaipis.org/ wp-content/uploads/2015/02/Los-medios-de-comunicacio-n-y-el-suicidio.pdf.

Philips, D. (1974). The Influence of Suggestion on Suicide: Substantive and Theoretical Implications of the Werther Effect. American Sociological Review, 39(3), 340-354.

Rímola, A. (2015). Prevención del suicidio. Ministerio de Salud de Costa Rica. https://www.paho.org/hq/ dmdocuments/2015/rimola-suicide-prevention.pdf

Ruder, T., Gary, M., Ampanozi, G., Thali, M., \& Fischer, N. (2011). Suicide Announcement on Facebook. Crisis, $32(5), 280-282$.

Sabariego, M., Massot, I., \& Dorio, I. (2009). Métodos de Investigación Cualitativa. En R. Bisquerra (Ed.), Metodología de la investigación educativa (pp.293-328). Editorial La Muralla S.A.

Solano, P., Ustulin, M., Pizzorno, E., Vichi, M. Pompili, M., Serafini, M., \& Amore, M. (2016). A Google-based approach for monitoring suicide risk. Psychiatry Research, 246, 581-586.

Swanson, S., \& Colman, I. (2010). Association between exposure to suicide and suicidality outcomes in youth. CMAJ, 185(10) 870-877. 
Sobre el autor y las autoras:

Recibido: 06 de Agosto de 2019

Revisión recibida: 23 de Octubre de 2020

Aceptado: 13 de Noviembre de 2020

Ricardo Millán González es especialista en Psiquiatría por la Universidad de Costa Rica, especialista en Psiquiatría de Interconsulta y Enlace de la Pontificia Universidad Javeriana de Bogotá, Colombia y máster en Ciencias Neurológicas por la Universidad de Costa Rica. Se desempeña como profesor asociado de la Escuela de Medicina de la Universidad de Costa Rica y fue el editor del Manual de Exploración y Semiología Psiquiátrica, publicado en 2019 por la Biblioteca Nacional de Salud y Seguridad Social (BINASSS). Actualmente, desarrolla trabajos de investigación en depresión en atención primaria y en epidemiología de la enfermedad de Parkinson, además de investigación clínica. Trabaja como profesor de posgrado del curso de Psiquiatría de Interconsulta y Enlace para la especialidad de Psiquiatría del Centro de Desarrollo Estratégico e Información en Salud y Seguridad Social (CENDEISSS)/Universidad de Costa Rica (UCR), y previamente fungió como profesor del posgrado de la especialidad de Psicología Clínica del CENDEISSS/UCR. Es miembro de la European Association of Psychosomatic Medicine desde 2011 y de la Academy of Consultation-Liaison Psychiatry desde 2019. Ejerce la práctica privada en el Hospital Clínica Bíblica desde 2012.

Cynthia Córdoba López es bachiller, licenciada en Psicología y máster en Psicología clínica y de la salud de la Universidad de Costa Rica por la Universidad de Costa Rica. Obtuvo el Máster en Administración de Servicios de Salud Sostenible con énfasis en Gerencia de la Salud de la Universidad Estatal a Distancia. Es especialista en Monitoreo y Evaluación para la Gestión de Políticas y Programas de VIH y sida con énfasis en Economía de la Salud y VIH (Diplomado Profesional) por el Instituto Centroamericano de Administración Pública. Labora como funcionaria de la Universidad de Costa Rica, donde coordina el área de Psicología en la Oficina de Bienestar y Salud. Ha colaborado como docente en los cursos de investigación de la carrera de Psicología de la Universidad Nacional. Actualmente, coordina el Plan de Salud Mental 2020 dirigido a la población estudiantil y laboral de la Universidad de Costa Rica.

Marjorie Moreno Salas es bachiller y licenciada en Psicología por la Universidad de Costa Rica. Egresó de la Maestría en Terapia Familiar de la Universidad Libre de Costa Rica; se desempeña como especialista en Psicología Clínica de la Universidad de Costa Rica y doctora en Ciencias de la Educación de la Universidad Católica de Costa Rica. Labora como docente del Sistema de Estudios de Posgrado de la Universidad Estatal a Distancia. Es funcionaria de la Caja Costarricense de Seguro Social destacada en el Hospital Nacional Psiquiátrico Dr. Roberto Chacón Paut. Adicionalmente, labora en atención psicológica a nivel privado. También, forma parte como miembro fundadora de la Asociación Costarricense de Psicotrauma (ACOTRAUMA) y de la Asociación Costarricense de Terapia Breve Estratégica, (ACOTEBE). Actualmente, ejerce el cargo de la vicepresidenta de ambas asociaciones dedicas al estudio, capacitación e investigación de dichas materias. Cuenta con publicaciones a nivel nacional e internacional sobre diversos temas de su disciplina. 